\title{
Variability Among Forecast Models for the Apple Sooty Blotch/Flyspeck Disease Complex
}

\author{
Daniel R. Cooley, Department of Plant, Soil, \& Insect Sciences, University of Massachusetts, Amherst; David A. Rosenberger, Hud- \\ son Valley Lab, Cornell University, Highland, NY; Mark L. Gleason, Department of Plant Pathology, Iowa State University, Ames; \\ Glen Koehler, Pest Management Office, University of Maine, Orono; Kerik Cox, Hudson Valley Lab, Cornell University, Highland, \\ NY; Jon M. Clements, Department of Plant, Soil, \& Insect Sciences, University of Massachusetts, Amherst; Turner B. Sutton, Depart- \\ ment of Plant Pathology, North Carolina State University, Raleigh; Angela Madeiras, Department of Plant, Soil, \& Insect Sciences, \\ University of Massachusetts, Amherst; and John R. Hartman, Department of Plant Pathology, University of Kentucky, Lexington
}

\begin{abstract}
Cooley, D. R., Rosenberger, D. A., Gleason, M. L., Koehler, G., Cox, K., Clements, J. M., Sutton, T. B., Madeiras, A., and Hartman, J. R. 2011. Variability among forecast models for the apple sooty blotch/flyspeck disease complex. Plant Dis. 95:1179-1186.

Several disease forecast models have been developed to guide treatment of the sooty blotch and flyspeck (SBFS) disease complex of apple. Generally, these empirical models are based on the accumulation of hours of leaf wetness (leaf wetness duration [LWD]) from a biofix at or near the phenological growth stage petal fall, when apple flower petals senesce and drop. The models recommend timing of the initial fungicide application targeting SBFS. However, there are significant differences among SBFS forecast models in terms of biofix and the length of LWD thresholds. A comparison of models using a single input data set generated recommendations for the first SBFS fungicide application that differed by up to 5 weeks. In an attempt to improve consistency among models, potential sources for differences were examined. Leaf wetness (LW) is a particularly variable parameter among models, depending on whether on-site or remote weather data

were used, the types of sensors and their placement for on-site monitors, and the models used to estimate LW remotely. When SBFS models are applied in the field, recommended treatment thresholds do not always match the method of data acquisition, leading to potential failures. Horticultural factors, such as tree size, canopy density, and cultivar, and orchard site factors such as the distance to potential inoculum sources can impact risk of SBFS and should also be considered in forecast models. The number of fungal species identified as contributors to the SBFS disease complex has expanded tremendously in recent years. A lack of understanding of key epidemiological factors for different fungi in the complex, and which fungi represent the most challenging management problems, are obvious issues in the development of improved SBFS models. If SBFS forecast models are to be adopted, researchers will need to address these issues.
\end{abstract}

Sooty blotch and flyspeck (SBFS), a disease complex caused by numerous fungal species, impacts pome fruit production in humid regions worldwide $(4,5,18,21,35,58,60)$. Although the disease is limited to waxy cuticles and does little damage to trees or fruit, the blemishes it causes can substantially decrease apple fruit quality and, therefore, value. In the eastern production regions of the United States, growers generally apply from 30 to $50 \%$ of a season's fungicides, usually from four to eight applications, to manage a set of summer diseases, including SBFS, black rot (Botryosphaeria obtusa (Schwein.) Shoemaker), bitter rot (Glomerella cingulata (Stoneman) Spauld. \& H. Schrenk), white rot or bot rot (B. dothidea (Moug.) Ces. \& De Not.), Brooks fruit spot (Mycosphaerella pomi (Pass.) Lindau), and Alternaria blotch (Alternaria mali Roberts) (54). SBFS is the only consistent summer disease problem in northern regions such as New England but the other diseases become increasingly important going from north to south.

Calendar-based management programs of applications at 1- to 3week intervals for SBFS are common because the etiology and epidemiology of the disease are not well understood. However, this prophylactic approach may result in over-application of fungicides

Corresponding author: Daniel Cooley,

E-mail: dcooley@microbio.umass.edu

Accepted for publication 9 May 2011.

doi:10.1094/PDIS-03-11-0248

(C) 2011 The American Phytopathological Society or, in some years, result in late-season outbreaks of disease, the result of unprotected late-season infections arising because fungicide applications are stopped too soon or there is unusually frequent or abundant late-season precipitation $(45,46)$. To better time fungicide sprays, several SBFS risk forecast models have been developed $(3,11,16,25,43,55)$. These SBFS models are empirical, built by identifying environmental variables that best predict the first appearance of signs on unprotected apple fruit. They measure accumulated leaf wetness duration in hours (LWD) as the primary parameter, except in the upper midwestern United States, where periods of relative humidity $(\mathrm{RH}) \geq 97 \%$ have been determined to be a more accurate predictor (16). LWD is measured from a biofix, usually at or near the time of flower petal fall (PF). When a critical LWD value is reached, a fungicide spray is recommended in order to prevent, eradicate, or temporarily stop disease development (43). As a result, one to four fungicide applications that would normally be made during the weeks after PF on a calendar schedule may be eliminated without sacrificing disease control. Some models also evaluate risk of subsequent infections $(33,43,55)$.

Beyond this general format of SBFS risk forecast models, there is considerable variability in the way each model is constructed and applied. A comparison of different SBFS models used in the eastern United States shows they may differ in terms of biofix, the LWD used to predict the first appearance of signs, the criteria for determining LWD, and recommended treatment (Table 1). In addition, users may obtain weather data for use with the models in several ways, including on-site mechanical recorders, electronic weather stations, or web-based remote weather services $(39,53)$. Leaf wetness (LW), in particular, varies widely depending on 
which weather-monitoring method is used and how it is employed (22), yet little attention has been given to matching weather monitoring methods to particular models, which may lead to inappropriate use of SBFS models. In this review, our purpose is to outline the extent of variation between various SBFS models, demonstrate how this variability may impact management decisions, and suggest ways that the models may be improved and more effectively used.

SBFS forecast models. The first SBFS forecast model. Brown and Sutton developed the first SBFS model in North Carolina, taking weather data from 1987 to 1994 and comparing it with the first appearance of SBFS on fruit of unsprayed 'Golden Delicious' trees (11). They estimated a predictor for the first appearance of SBFS signs of 273 LWD measured from 10 days after PF, with only those wetting periods that were $4 \mathrm{~h}$ or longer counted. Over the 7 years evaluated, SBFS first appeared between 209 and 310 LWD. Based on this finding, they recommended applying a benzimidazole fungicide, such as benomyl (Benlate) or thiophanate methyl (Topsin M), at a treatment threshold of 200 to 225 LWD to eradicate SBFS.

To measure LW, Brown and Sutton used a device based on a cotton fiber string, a de Wit LW meter (I. F. G. de Wit, Roden, The Netherlands). The device was placed on the north side of an apple tree, inside the drip line, $1.5 \mathrm{~m}$ above the ground. Brown and Sutton said a deflection of $50 \%$ or more of the pen on the recording chart indicated LW (10). They concluded that "...the threshold that we have established with the de Wit sensor may have to be modified if other sensors are used", and noted that an adaptation of their model in Kentucky using an electronic sensor worked using a lower threshold. From the outset, it was noted that using an electronic sensor with a forecast model developed using a mechanical sensor like the de Wit can be problematic.

Revising the North Carolina model. Hartman and Smigell tested the Brown/Sutton model in Kentucky and adjusted the treatment threshold to work with an electronic LW sensor $(25,49,50)$. They adapted a technique first used to study SBFS by Hickey et al. (27), wherein fruit are covered by bags for varying periods during the season to determine when fruit were first infected by SBFS. At regular time intervals of approximately 1 week, fruit were arbitrarily bagged. Fruit bagged before 175 LWD measured from 10 days after PF did not develop SBFS. Based on this, the researchers recommended a 175-LWD treatment threshold. In addition to reducing LWD, the revised model counted all wetting hours. Using the revised model, Hartman effectively controlled SBFS in trials, saving between two to four fungicide applications relative to calendarbased prophylactic sprays.

This Hartman/Smigell revision of the Brown/Sutton model was tested in three states in the upper midwestern United States in 2001-02 in both university trials and commercial orchards $(3,20)$.
In addition to testing the relative efficacy and application efficiency of the forecast model versus conventional cover sprays, the study compared on-site weather measurement with site-specific remote estimates supplied by SkyBit (ZedX Inc., Bellefonte, PA) $(23,47)$. The blocks sprayed using model recommendations generally required fewer fungicide applications than the blocks sprayed on a calendar basis but the model-managed plots often had significantly higher incidence of SBFS. The study found that SkyBit overestimated LW relative to electronic sensors placed in apple canopies, meaning that the treatment threshold was reached sooner in SkyBit blocks.

Gleason and colleagues $(6,16)$ suggested that, during the growing season, the midwestern United States is significantly drier than the southeastern United States. Therefore, while rain events provide the bulk of the leaf wetting periods measured in the southeastern United States, high humidity and dew provide most of the LWD recorded in Iowa and Wisconsin. After examining other possible weather variables, these researchers found that accumulated periods of $\mathrm{RH} \geq 97 \%$ provided better forecasts than LWD in the midwestern United States, though the same study confirmed that LWD performed better than humidity in the southeastern United States.

Adaptation of the North Carolina model to the northeastern United States. In the northeastern United States, Rosenberger developed an SBFS forecast model that was based on the Brown/Sutton model and incorporated fungicide depletion (43). Rosenberger's model computes the timings for both the first summer fungicide and later fungicides, and was based on using a string-activated wetness sensor in the same way as described for the Brown/Sutton model. According to the model, the first fungicide targeting SBFS should be applied at 270 LWD from PF (rather than $\mathrm{PF}+10$ as in the Brown/Sutton model); subsequent fungicides should be applied so as to allow no more than an additional 270 LWD when fruit are unprotected by fungicide, counting from the first SBFS spray and continuing to harvest. To use this approach, the length of time fungicides are effective must be known. Different fungicides have different effective periods, which Rosenberger (41) categorized in three groups based on time or rainfall from the most recent application: (i) 21 days or $6.4 \mathrm{~cm}$ of rainfall, (ii) 21 days or $5.1 \mathrm{~cm}$ of rainfall, and (iii) 14 days or $3.8 \mathrm{~cm}$ of rainfall.

This SBFS model is based on the hypothesis that inoculum develops on reservoir hosts adjacent to orchards and disseminates to infect fruit. SBFS inoculum is assumed to be negligible in a well-sprayed commercial orchard where SBFS was not present on apple fruit the previous year. Relatively small amounts of inoculum may be dispersed to apple fruit when ascosporic inoculum is released during several weeks near PF but fungicide applications targeting apple scab and powdery mildew prevent the SBFS spores

Table 1. List of major sooty blotch and flyspeck models describing the type of action being recommended, the biofix, the weather parameter used in calculating the threshold, the point at which first signs appear, and the treatment threshold ${ }^{t}$

\begin{tabular}{|c|c|c|c|c|c|}
\hline Model & Biofix & LWD & First signs & $\begin{array}{l}\text { Treatment } \\
\text { threshold }\end{array}$ & Recommended action \\
\hline Brown/Sutton & 10 days after $P F$ & $\mathrm{LW} \geq 4 \mathrm{~h}$ & $273 \mathrm{~h}$ & $200-225 \mathrm{~h}$ & First benzimidazole fungicide \\
\hline Brown/Sutton/Hartman & 10 days after $P F$ & All LW h & $218 \mathrm{~h}^{\mathrm{u}}$ & $175 \mathrm{~h}$ & First fungicide \\
\hline Gleason/Duttweiler & Date of last scab fungicide & RH periods $\geq 97 \% \mathrm{~h}$ and $\geq 4 \mathrm{~h}$ & $192 \mathrm{~h}$ & $192 \mathrm{~h}$ & First fungicide \\
\hline Rosenberger & $\mathrm{PF}$ & All LW h ${ }^{v}$ & $540 \mathrm{~h}$ & $270 \mathrm{~h}^{\mathrm{v}}$ & First fungicide and follow-up \\
\hline Orchard Radar & $\mathrm{PF}$ & All LW h, temp. adj. & $424 \mathrm{~h}^{\mathrm{w}}$ & $212 \mathrm{~h}^{\mathrm{w}}$ & First fungicide and follow-up \\
\hline NEWA & $\mathrm{PF}$ & All LW h & $200 \mathrm{~h}$ & $175 \mathrm{~h}^{\mathrm{x}}$ & First fungicide and follow-up \\
\hline Skybit & $\mathrm{PF}$ & All LW hy & $?$ & $350 \mathrm{~h}$ & First fungicide \\
\hline SpecWare & $\mathrm{PF}$ & $L W \geq 3 \mathrm{~h}$ & ? & $250 / 300 \mathrm{~h}^{\mathrm{z}}$ & First fungicide \\
\hline
\end{tabular}

${ }^{\mathrm{t}} \mathrm{LWD}=$ leaf wetness duration, $\mathrm{PF}=$ Petal fall, $\mathrm{LW}=$ leaf wetness, $\mathrm{RH}=$ relative humidity, temp. adj. = temperature adjusted, and NEWA $=$ Network for

Environmental and Weather Applications.

" Average of the reported range, 185 to $251 \mathrm{~h}$.

${ }^{v}$ As measured with a de Wit string-activated recording chart. Assumes application of at least one post-PF fungicide targeting scab.

${ }^{\mathrm{w}}$ Calculated based on Rosenberger thresholds but adjusted for temperature.

${ }^{\mathrm{x}}$ Measured electronically; interpolated from original de Wit measurements.

${ }^{y} \mathrm{LW}$ is estimated from RH, wind speed, and other data.

${ }^{\mathrm{z}}$ For southern/northern orchards. 
from establishing colonies on fruit. On reservoir hosts, however, SBFS fungi can develop and produce abundant amounts of inoculum. Rosenberger theorized that the 270 LWD from PF to development of SBFS signs identified in North Carolina reflects the period necessary for SBFS inoculum to develop to economically significant levels on reservoir hosts in the Northeast. After the initial 270 LWD, Rosenberger (43) estimated that an additional 270 LWD is needed for inoculum that lands on fruit to develop signs. Hence, if fruit is unprotected by fungicide after scab applications have stopped, it will take a total of 540 LWD from PF for SBFS signs to develop. At any time when SBFS fungi are present on fruit, growth can be stopped by a fungicide application, but such applications may be fungistatic, not fungicidal $(41,42)$. After a given fungicide application is depleted by some combination of rainfall, tissue growth, and oxidative and photochemical breakdown, SBFS fungi apparently resume growth through the incubation period. Once fruit have been unprotected for a total of 540 LWD from PF, SBFS signs appear. Recently, Rosenberger has suggested that, if electronic sensors are used, based on the Hartman/Smigell model, it may be more appropriate to use shorter thresholds of 175 LWD before the first fungicide application followed by a 175 LWD of unprotected fruit (D. A. Rosenberger, personal communication).

Development of a European SB model. Trapman has developed a model to manage SB in organic apple orchards in Europe, using an approach that evaluates risk of infection through the growing season and is a component of the proprietary RIMpro apple pest management decision support system (55). This model uses a biofix of $\mathrm{PF}+10$ days and assumes that inoculum originates primarily from within the orchard and is splash dispersed, fungal growth on fruit occurs when wet conditions are measured or $\mathrm{RH}$ is $>97 \%$, and symptoms first appear at $225 \mathrm{LWD}$ and fully develop after 325 LWD. Growth is adjusted for temperature (29), and a proportion of spores are assumed to die during dry periods. Wetting is measured electronically. In a preliminary test, post-infection treatments of lime sulfur or coconut soap provided 72 to $100 \%$ control (55).

Delivery of SBFS forecasts. Written recommendations. Users may track weather data and can use written recommendations such as those in The New England Tree Fruit Management Guide (12). This guide uses a 270 LWD from PF as the threshold for the first SBFS fungicide application.

Spectrum WatchDog and SpecWare. The Spectrum WatchDog weather station is offered with a bundle of pest forecasting software, SpecWare (Spectrum Technologies, Inc., Plainfield, IL). The documentation for the SBFS model in the software package (1) describes the SBFS complex as caused by three pathogens. Apparently the SpecWare model is based on 1996 recommendations made by Jones and Sutton (30), though there are differences between this source and the model as described in the manual. Jones and Sutton use a biofix of 10 days after PF and the model is used to recommend when the first SBFS fungicide should be applied. The Spectrum model uses a PF biofix and has two infection thresholds, one for "Southern States" at 250 LWD and one for "Northern States" at $300 \mathrm{LWD}$, although a specific listing of such states is not provided. After a threshold is reached, any 3-h wetting period is considered sufficient for SBFS infection. The 300-LWD threshold is probably based on Jones' interpretation of earlier work done by Rosenberger that suggested that a threshold of 300 LWD could be used with benzimidazole fungicides (41). Therefore, the SpecWare model is dated, although the first application can safely be delayed until 300 LWD from PF, so long as one recognizes that this approach may allow some establishment of SBFS on apple fruit that must thereafter be suppressed by maintaining an effective fungicide residue on the fruit.

SkyBit. SkyBit estimates weather variables for a specific site based on publically available weather data. Using proprietary algorithms, SkyBit estimates weather at a resolution of $1 \mathrm{~km}^{2}$ (31). Users supply SkyBit with the precise latitude, longitude, and elevation of a site and, for a subscription fee, receive information on past weather, weather forecasts, and risk evaluations for various pests based on models. SkyBit does not require maintenance of a weather station on site, and generally performs as well as on-site equipment $(22,38)$. The SkyBit SBFS model is based on the Hartman/Smigell model. For purposes of the SBFS model, SkyBit uses a 350-LWD threshold rather than 175 LWD, suggesting that either LWD is overestimated or that the model is designed to allow a longer spray interval after PF on the assumption that SBFS infections that may occur between 175 and 350 LWD from PF will be suppressed by subsequent fungicide applications.

Orchard Radar. Orchard Radar is a web-based pest management system for New England (33) that has been operating since 1997 (Pest Resources Online, New England, University of Maine, Orono). This system uses weather data from SkyBit and, for 10day predictions, uses SkyBit forecasts. For predictions beyond 10 days, it uses the 30-year averages of historical weather data for a site. Individuals contract with SkyBit to send data to the University of Maine, where it is used in spreadsheet-based integrated pest management (IPM) models. For SBFS, Orchard Radar is based on the Rosenberger model, except that LW data are adjusted for temperature. Other researchers also have suggested that temperature plays a role in SBFS development (56). Using information on the depletion of fungicide residue from Rosenberger (43), Orchard Radar provides growers with information on when the protection from that application should be depleted (protection end date) and an estimate of when SBFS signs will first appear if no further fungicides are applied. Three estimates are provided, the first being a worst-case prediction for the wettest $20 \%$ of years, the second based on SkyBit and historical average data, and the third a prediction intended to estimate possible timing of SBFS sign appearance on apple fruit grown in small, well-pruned trees on a site with good air movement that is a substantial distance from SBFS reservoir plants.

Network for Environmental and Weather Applications. The Network for Environmental and Weather Applications (NEWA), a collaborative effort of the New York State IPM Program and the Northeast Regional Climate Center at Cornell University, Geneva, NY, delivers IPM information for several crops and obtains weather data from private weather stations and airport weather stations (2). The NEWA SBFS risk prediction module relies primarily on a simplified Rosenberger model, except that it uses threshold values of 175 LWD from PF rather than 270 LWD, to account for the fact that LW data used by NEWA is largely from electronic LW sensors. NEWA tracks LW from PF and asks growers to input the date of the most recent fungicide application. It then uses time and rainfall to estimate fungicide depletion and assigns three levels of risk (low, moderate, and high) based on LWD from PF and fungicide depletion.

RIMpro. The RIMpro model collects data from weather stations in or immediately adjacent to orchards. Data are processed on location by a personal computer using RIMpro software, and output is shown as risk of infection on a 0 -to-1,000 scale, with dates when appearance of SB signs would be expected, with their relative severity. The SB model in RIMpro is being tested in European trials (21).

Problems associated with measuring LW. The problem of measuring LW has been extensively studied $(22,28,34,48,53)$. However, LW has never been quantitatively defined, and its measurement is problematic (37). In the descriptions above, it is obvious that measurement of LW can be highly variable, depending on the type of instrument used, if any, and where sensors are placed. For example, LWD based on five different sources, including an Onset Hobo U30 weather station (Onset Computer Corp., Pocassett, MA) with the LW threshold set to $40 \%$ of full range, the same weather station with the LW threshold set at $100 \%$ of full range, a Spectrum weather station with the threshold at $40 \%$ of full range, Skybit, and an estimate based on airport weather data using a fuzzy logic algorithm (32) were compared at the University of Massachusetts Cold Spring Orchard Research and Education Center, Belchertown for the period 3 May to 12 June 2010. At this location, by 12 June, the largest estimate of LWD is over two and a half times greater than the smallest (Fig. 1). 
Although the differences are very large, the actual relationship between the different measurements is relatively constant. That infers that the estimates are acceptable as long as they are used with an appropriate model and threshold. For example, a user should not use SkyBit weather data with a threshold developed using a string LW sensor.

In commercial applications, little attention has generally been paid to standardizing the height of an LW sensor above the ground, the direction it should face, or whether it should be placed at a specific angle, though these factors can significantly affect LW measurements (34). Recently, consensus has built around facing sensors north at a $45^{\circ}$ angle relative to level (22).

The most common recommendation for placement of LW sensors is to take measurements in the canopy of a typical tree in an orchard. However, placing sensors in tree canopies can lead to practical problems because pesticides and other chemicals sprayed in an orchard can corrode the electronics in LW sensors. To avoid damaging sensors and ease access to the instruments, it would be useful to place sensors near but not in the orchard. To standardize environmental data, both researchers and growers should consider rules for placing sensors. For example, in addition to the rules for sensor angle and direction, other considerations might include placement over mowed grass, at least $10 \mathrm{~m}$ from any building or other physical features that could inhibit air circulation or effect microclimate. Inconsistency in LW measurement also may occur if sensor wetness thresholds are set too low or too high. Even with standardization in placing and calibrating equipment, there is a great deal of variability between sensors (38).

LW measurement is so variable that several researchers have recommended using other, more easily obtained variables that are quantitatively defined $(22,37,38)$. However, in one experiment, while accumulating hours with $\geq 97 \%$ RH provided a better forecast of SBFS infections than LWD in the upper midwestern United States, LWD was clearly better than the RH threshold in the southeastern United States (16), indicating that simply substituting RH for LW does not provide the best forecasts in all cases.

Publically available weather information that is used in off-site systems generally does not include LW data; therefore, if LW is

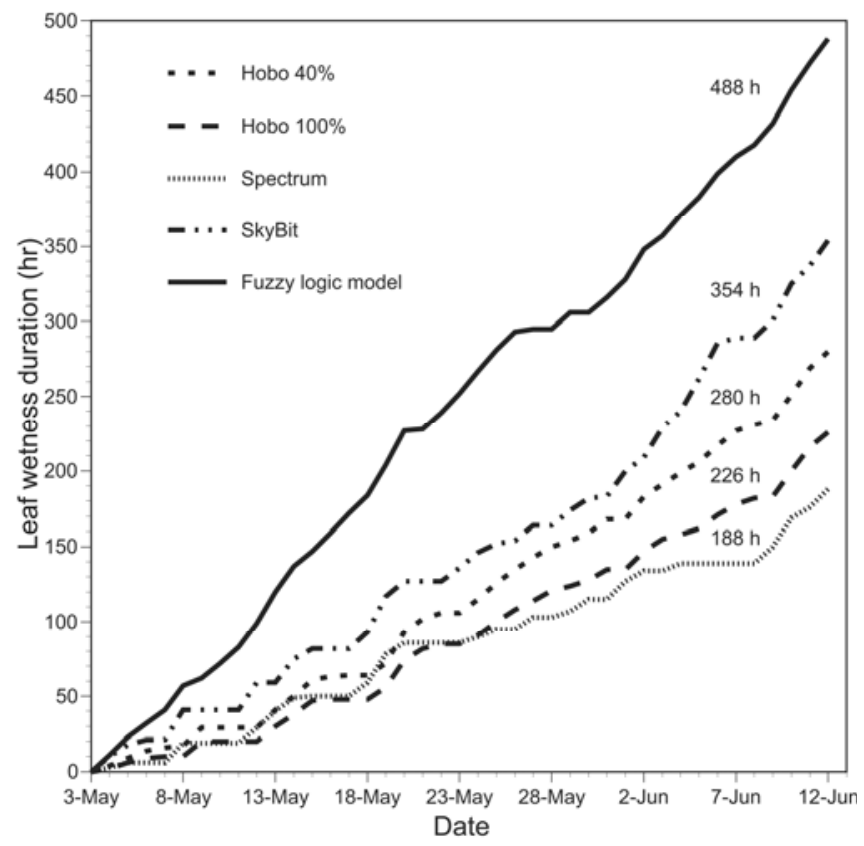

Fig. 1. Leaf wetness duration (LWD) at the University of Massachusetts Cold Spring Research and Education Center, Belchertown, in 2010, measured using five different methods: an Onset Hobo electronic leaf wetness sensor using a $40 \%$ threshold and a $100 \%$ threshold, a Spectrum WatchDog electronic leaf wetness sensor using a $40 \%$ threshold, SkyBit remote estimates, and remote estimates from a fuzzy logic leaf wetness model (32). LWD on 12 June for each method is shown next to the method's respective graph line on the right of the chart. needed in a forecast model, it must be estimated based on other available data such as temperature, RH, and wind speed (32). Such models for determining LW ultimately introduce complexity to forecast models, and it is not clear that they always add substantial value compared with a more readily obtainable variable such as $\mathrm{RH}$ or dew-point deficit.

Ultimately, if LW is used regardless of the measurement method used, each specific disease model must use the LW measurement appropriate to it. To take one LW measurement method and apply it to a model developed with a different method will lead to errors. For example, Babadoost et al. (3) applied the Brown/Sutton/ Hartman model and compared on-site weather stations with SkyBit data (not the SkyBit model). Because SkyBit apparently accumulates LW hours faster than on-site equipment, using SkyBit data with the 175-LWD threshold meant that fungicides were applied much sooner on SkyBit-based blocks compared with blocks timed by on-site equipment. Although SkyBit has suggested that their 350 LWD is equivalent to 270 LWD measured by a de Wit monitor or 175 LWD measured by an electronic instrument, to our knowledge, the 350-LWD threshold has not been tested in field trials in conjunction with an appropriate model to determine its performance.

In-field comparison of SBFS models. Given the considerable potential for variability between different models, we compared five models used in the eastern United States to make SBFS recommendations. The comparisons were made by monitoring environmental data at the University of Massachusetts Cold Spring Orchard Research and Education Center, Belchertown, using onsite and remote methods, and using that data in appropriate models to forecast an SBFS treatment threshold. The models included the 270-LWD threshold described in the New England Tree Fruit Guide (12) using data from a Hobo Data Logger with a threshold of $40 \%$ of the full range of the LW sensor to indicate "wetness", the SpecWare SBFS model using a Spectrum Watchdog weather station and a $40 \%$ response on the LW sensor, the SkyBit SBFS model using SkyBit weather data, the Orchard Radar forecasting system for SBFS, and the Cornell NEWA SBFS model using data from the on-site Hobo station.

Large differences were identified between the various recommendations in terms of the date of the first fungicide application for SBFS (Fig. 2). The earliest treatment recommendation was 2 June (NEWA/Hobo) and the latest 17 July (SpecWare'Watchdog), a difference of over 6 weeks. The SpecWare recommendation was much later than any other and, if it was excluded, the range of the other four models went from 2 June to 16 June, 2 weeks.

Under normal conditions, the 6-week difference in time would result in one to three fewer fungicide applications for the later treatment recommendation relative to the earlier ones. The 2week difference might result in one extra fungicide application in the earlier treatment. No treatments were made to test field performance of the models; therefore, no conclusions can be made as to which recommendations were most accurate. However, the differences clearly show that SBFS forecast models need improvement.

Developing improved SBFS models. Errors in SBFS forecasts may occur as the result of errors in collecting weather data, particularly LW, and from misapplying the empirical models used to evaluate that data. The accuracy of SBFS models and all weather-based disease forecasting models may be improved if LW measurement were better standardized, both at the time of model development and when models are implemented by growers. In the case of SBFS, for example, there is a need to confirm whether a 270-LWD threshold is equivalent to a 175-LWD threshold measured on a typical electronic sensor. Researchers have pointed out that there is no single "best" method to acquire weather data for use in disease-warning systems (22) but growers, consultants, and researchers should make a concerted attempt to ensure that data are being applied appropriately; specifically, that environmental measurement methods, particularly for LW, are appropriate to the forecast method used. 
A better understanding of SBFS epidemiology also would improve forecast models. Specifically, it would be useful to know when inoculum is mature and able to initiate infections on fruit, the environmental conditions that lead to fruit infection (e.g., wetting, high humidity, or temperature), and the amount of time as related to these environmental factors required for infections to develop into signs on fruit. This will probably mean developing separate models for inoculum development and for sign development, just as there are separate models for these processes in apple scab management $(19,36,51,52)$. Although Rosenberger's model (43) suggests that LWD is related first to inoculum development and later to sign development, the impact of LWD on inoculum development remains unknown.

In North Carolina, SB infections were initiated 10 to 21 days after PF (10). Bagging studies in the United States have shown that fruit left unprotected by bags for up to several weeks immediately after PF did not develop SBFS (25-27). However, other bagging studies have concluded that SBFS fungi can colonize fruit at any time during its development (21).

The time from putative inoculation to SBFS sign development varies by location and year; in North Carolina (11) at a single site, the first appearance of SBFS signs occurred from 40 to 89 days after PF, depending on the year. In Poland, the incubation period was from 29 to 45 days (24). Rosenberger has observed that development of signs on fruit collected from the field can be accelerated by incubating harvested apple samples in controlled conditions of high humidity (essentially 100\%) at normal ambient temperatures $\left(21^{\circ} \mathrm{C}\right)(44)$. For example, one test fruit on trees in the field had $15.5 \%$ FS incidence on 31 August, whereas fruit that had been harvested 2 weeks earlier and incubated had $82.5 \%$ FS incidence. If sign development depends on high $\mathrm{RH}$, in situ, the length of time from inoculation to sign development would be expected to vary depending on the frequency of periods of high RH. Hence, because

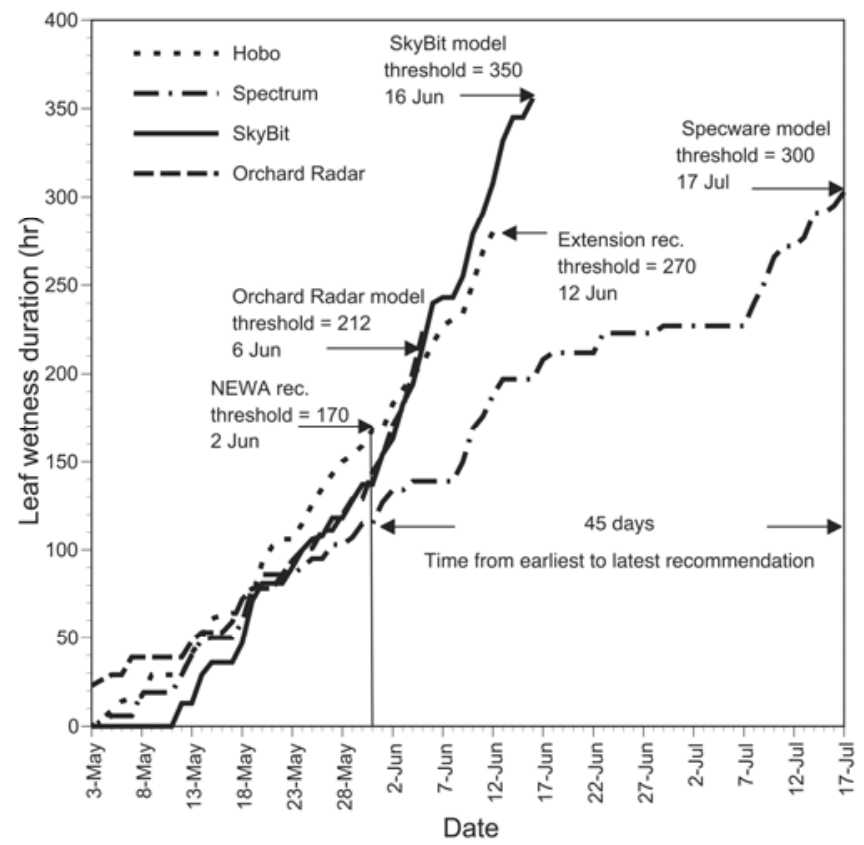

Fig. 2. Recommended dates for the first fungicide application targeting sooty blotch and flyspeck as determined by five models based on conditions at the University of Massachusetts Cold Spring Orchard, Belchertown in 2010. Methods include leaf wetness duration in hours (LWD) measured using an Onset Hobo weather station with the leaf wetness threshold set at $40 \%$ of full response and a 270 LWD threshold recommended by Extension; the Cornell Network for Environmental and Weather Applications model using a 170 LWD threshold and the same weather station data; the SkyBit model using mesoscale data for the Belchertown site from Zedex, Inc. and a 350 LWD threshold; the New England Orchard Radar model using the same data from SkyBit and a 212 temperature adjusted LWD; and an onsite Spectrum weather station with a leaf wetness sensor threshold set at $40 \%$ of full range and the recommended 300 LWD treatment threshold. humidity and temperature fluctuate outside the optimal ranges for SBFS growth in the field, it generally takes much longer for SBFS signs to show on fruit there than under a continuous regimen of very high $\mathrm{RH}$, though infections are undoubtedly present. In one trial where most fungicide residues were removed in a single rain event in August, Rosenberger (44) noted a rapid increase in FS signs on fruit between 32 and 37 days after the rain. These observations and others support the hypothesis that there is a period of cryptic fungal development for SBFS fungi driven by periods of very high $\mathrm{RH}(\geq 95 \%)(14,16,29,40)$.

We have a general understanding of the epidemiology for one of the fungi in the SBFS complex, Schizothyrium pomi (Fig. 3). Work in Massachusetts has shown that inoculum development is highly correlated with temperature starting at green tip on 'McIntosh' (14). S. pomi ascospores start to develop near pink bud or bloom at 540 degree-days (base $0^{\circ} \mathrm{C}$ ) and ends approximately 3 to 4 weeks after PF at 1,625 degree-days (14). Hence, primary inoculum for flyspeck is available well before PF and continues to be available well after PF. In estimating the availability of FS inoculum, PF is not particularly relevant and using it as a biofix is arbitrary. It may be more accurate to use a green-tip biofix coupled with a degreeday-driven model to determine when inoculum is first available.

PF also can be imprecise because it does not occur in a single day but is a process that progresses over several days, from only a few flowers dropping petals to the point at which all flowers have lost their petals. PF is also highly dependent upon cultivar. For example, the original Sutton/Brown model was developed using Golden Delicious but is often applied in the Northeast using the McIntosh, which typically blooms and drops petals from 5 to 7 days before Golden Delicious growing at the same sites. Just as LW data should be appropriate to a specific model, if a PF biofix is
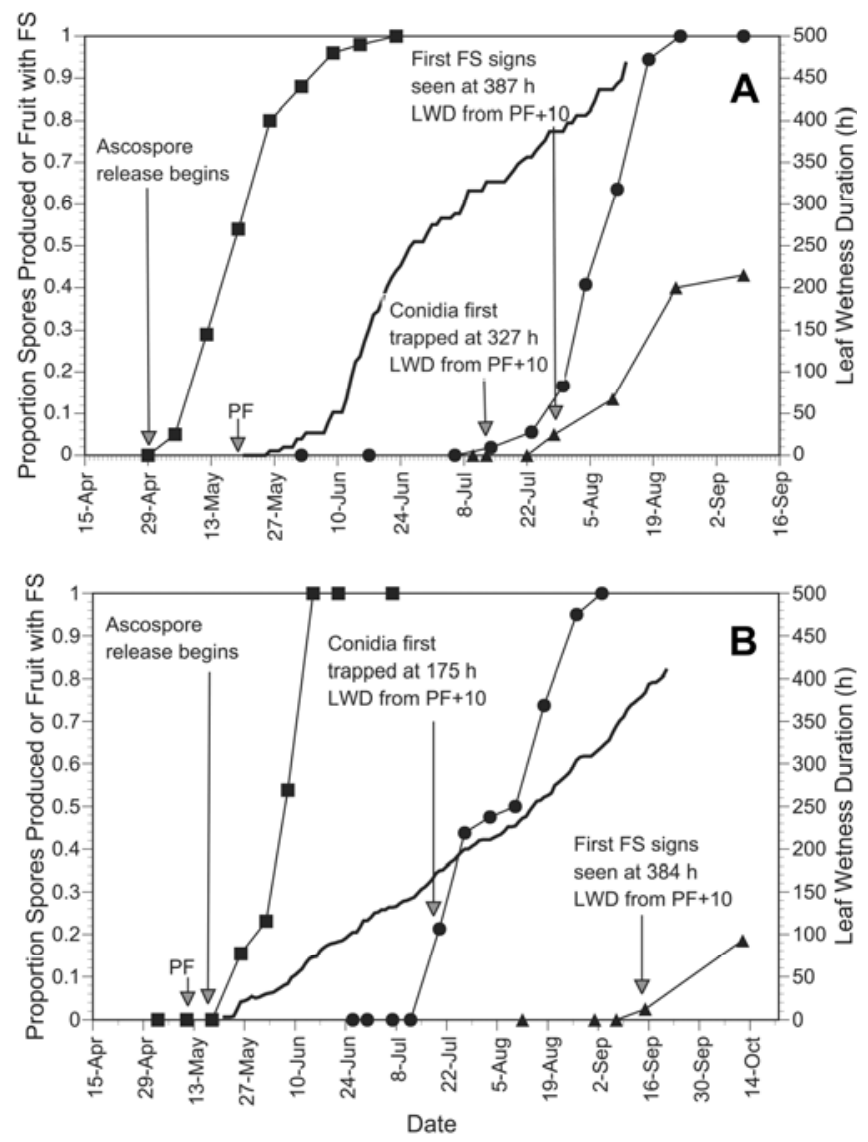

Fig. 3. Flyspeck epidemics in commercial orchards in A, Ashfield, MA, in 1998 and B, Shelburne, MA, in 1999. Accumulated percent mature Schizothyrium pomi ascospores (घ), accumulated percent $S$. pomi conidia $(\bullet)$, and percent fruit with flyspeck signs $(\mathbf{\Lambda})$ are presented, as well as hours leaf wetness duration (LWD; no symbol). 
used, it should be defined in terms of cultivar and the percentage of petals that have dropped.

Evidence suggests that secondary inoculum-specifically, conidia of the anamorph of $S$. pomi-may develop on reservoir hosts before fruit formation but that it does not move into orchards and infect until several days to several weeks after fruit set. Flyspeck epidemics from two orchards in Massachusetts are shown in Figure 3. In this case, the maturity of $S$. pomi ascospores from thyriothecia on blackberry canes (Rubus allegheniensis) growing next to a commercial orchard was monitored using squash mounts (14). Rotorod spore samplers (Sampling Technologies, St. Louis Park, MN) adjacent to the blackberry plants were used to sample $S$. pomi conidia. Apple trees near the blackberry plants were not sprayed with fungicides after PF and were monitored weekly for SBFS signs. Weather data were collected using a Campbell CR10X data logger (Campbell Scientific, Inc., Logan, UT) equipped with a temperature sensor and a painted printed-circuit wetness sensor mounted facing north at an angle $45^{\circ}$ from horizontal (6,34), located approximately $1.5 \mathrm{~m}$ over unmown grass at the edge of each orchard. The sequential pattern in terms of the type of inoculum produced in the epidemics is similar in both instances, with a discrete period of ascospore maturation followed by a period of conidial release, and then appearance of FS signs on fruit. It took 387 and 384 LWD measured from 10 days after McIntosh PF $(P F+10)$ in 1998 and 1999, respectively, for the first FS signs to appear on fruit. Conidia were first trapped at 327 and 175 LWD PF + 10 in 1998 and 1999, respectively. In both years, conidia were not trapped until ascospore maturation had ended, and FS signs were not observed until FS conidia had been trapped. Similar data led Rosenberger to formulate his SBFS model (43). The epidemiology of other SBFS fungi remains largely unknown.

Cultivar can influence levels of SBFS on apple fruit $(8,9)$. One factor responsible for cultivar differences may be the amount of material that leaches from fruit through epicuticular waxes. This hypothesis is supported by the finding that growth of SBFS fungi is stimulated by increases in fructose and sucrose on the surface of apple fruit (59) or when apple juice is included in in vitro cultures $(7,8)$. Cultivars also differ in their retention of nondeveloping fruitlets, so-called mummies, and it has been suggested that these mummies can serve as a significant source of SBFS inoculum (21). The time it takes fruit to mature also indirectly influences SBFS incidence. In replicated plantings of 45 apple cultivars in Massachusetts, New York, and Virginia, cultivars harvested earlier in the year tended to develop less SBFS than cultivars harvested later, presumably because the apple fruit that were harvested earlier did not have sufficient time in the orchard to develop signs (9). Within this study, Rosenberger controlled for harvest date and showed that significant cultivar differences still occurred (Table 2). For these reasons, it may be worth including cultivar as a variable in SBFS forecast models.

Tree architecture, distance to inoculum sources, and other physical factors in orchards can affect SBFS development $(17,57)$. In nonsprayed, fully dwarf apple trees in well-maintained orchards that are not near reservoir hosts in Ohio, SBFS signs did not develop until over $450 \mathrm{LWD}$, while larger trees with dense canopies growing near inoculum sources developed SBFS between 225 and 241 LWD (17). Differences in canopy density generate differences in microclimate (13) but these would not be reflected by weather sensors placed outside the canopy $(6,13)$. The relationship of SBFS incidence to orchard site factors, such as distance to reservoir hosts, the slope of the orchard site, and the amount of flyspeck observed on reservoir hosts adjacent to the orchard, has been studied (57). However, the coefficient of determination $\left(r^{2}\right)$ between individual site factors and SBFS on apple fruit, while sometimes significant, has never been large, usually in the range 0.10 to 0.20 . Thus, although orchard site factors probably play a role in determining SBFS incidence, these variables appear to be much less important than meteorological variables.

Additional studies may help to better define the critical points in SBFS epidemiology, determining whether LWD is the primary environmental driver of SBFS epidemics or whether another, more easily obtained variable, such as RH might be sufficiently predictive, or whether a biofix other than PF (or a set number of days thereafter) is more accurate. However, the SBFS complex is large, species composition varies from region to region (15), and the primary and secondary inoculum of many of the fungi in the com-

Table 2. Incidence and severity of sooty blotch and flyspeck (SBFS) on apple fruit from different cultivars harvested on three dates at Highland, NY, 2003

\begin{tabular}{|c|c|c|c|c|}
\hline \multirow[b]{2}{*}{ Cultivar } & \multirow[b]{2}{*}{ Harvest date } & \multirow[b]{2}{*}{ SBFS rating ${ }^{\mathrm{z}}$} & \multicolumn{2}{|c|}{ Disease incidence $(\%)^{\mathrm{y}}$} \\
\hline & & & FS & SB \\
\hline Ginger Gold & 21 August & $1.57 \mathrm{~b}$ & $91.9 \mathrm{~b}$ & $55.1 \mathrm{~b}$ \\
\hline Pristine & 21 August & nd & $89.8 \mathrm{~b}$ & $19.4 \mathrm{a}$ \\
\hline Sunrise & 21 August & $0.92 \mathrm{a}$ & $79.0 \mathrm{a}$ & $27.2 \mathrm{a}$ \\
\hline Yataka/M.9 & 11 September & 2.59 ef & $100.0 \mathrm{e}$ & $98.8 \mathrm{e}$ \\
\hline Arlet & 11 September & $1.99 \mathrm{~cd}$ & $90.8 \mathrm{~b}$ & $82.5 \mathrm{c}$ \\
\hline Gala Supreme & 11 September & $2.72 \mathrm{f}$ & $100.0 \mathrm{e}$ & $99.6 \mathrm{e}$ \\
\hline Golden Supreme & 11 September & $2.66 \mathrm{ef}$ & $100.0 \mathrm{e}$ & $100.0 \mathrm{e}$ \\
\hline Honeycrisp & 11 September & $2.16 \mathrm{~d}$ & 98.5 cde & $93.1 \mathrm{~cd}$ \\
\hline NY 75414 & 11 September & $1.81 \mathrm{bc}$ & $98.4 \mathrm{~cd}$ & $31.3 \mathrm{a}$ \\
\hline Senshu & 11 September & $2.43 \mathrm{e}$ & $100.0 \mathrm{e}$ & $100.0 \mathrm{e}$ \\
\hline Pioneer McIntosh & 11 September & $1.72 \mathrm{~b}$ & 99.3 de & $52.2 \mathrm{~b}$ \\
\hline Fulford Gala & 11 September & $1.65 \mathrm{~b}$ & $95.5 \mathrm{c}$ & $65.9 \mathrm{~b}$ \\
\hline Braeburn/M.9 & 9 October & $3.27 \mathrm{gh}$ & $100.0 \mathrm{e}$ & $83.2 \mathrm{~d}$ \\
\hline Golden Delicious/M.9 & 9 October & $3.50 \mathrm{~h}$ & $100.0 \mathrm{e}$ & $100.0 \mathrm{e}$ \\
\hline Creston & 9 October & $3.50 \mathrm{~h}$ & $100.0 \mathrm{e}$ & $100.0 \mathrm{e}$ \\
\hline Cameo & 9 October & $3.33 \mathrm{gh}$ & $100.0 \mathrm{e}$ & $100.0 \mathrm{e}$ \\
\hline Enterprise & 9 October & $2.49 \mathrm{e}$ & $100.0 \mathrm{e}$ & $99.2 \mathrm{e}$ \\
\hline Fuji & 9 October & $3.46 \mathrm{~h}$ & $100.0 \mathrm{e}$ & $100.0 \mathrm{e}$ \\
\hline Goldrush & 9 October & $4.00 \mathrm{i}$ & $100.0 \mathrm{e}$ & $100.0 \mathrm{e}$ \\
\hline Fortune & 9 October & $3.07 \mathrm{~g}$ & $100.0 \mathrm{e}$ & $100.0 \mathrm{e}$ \\
\hline Orin & 9 October & $4.01 \mathrm{i}$ & $99.6 \mathrm{de}$ & $99.6 \mathrm{e}$ \\
\hline Shizuka & 9 October & $3.90 \mathrm{i}$ & $100.0 \mathrm{e}$ & $100.0 \mathrm{e}$ \\
\hline Suncrisp & 9 October & $3.94 \mathrm{i}$ & $100.0 \mathrm{e}$ & $100.0 \mathrm{e}$ \\
\hline Mutsu & 9 October & $4.11 \mathrm{i}$ & $100.0 \mathrm{e}$ & $100.0 \mathrm{e}$ \\
\hline
\end{tabular}

x SBFS ratings or incidence levels followed by different letters within each column are significantly different as evaluated by Fisher's protected least significant difference, $P \leq 0.001$.

${ }^{y}$ Incidence of SB or FS as a percentage of sampled fruit (50 fruit per tree, five replicates per cultivar) based on the presence or absence of disease.

${ }^{\mathrm{z}}$ Rating of the side of an apple showing the most SBFS: $0=$ no SBFS, $1=<10,2=10$ to $25,3=25$ to $50,4=50$ to 75 , and $5=>75 \%$ surface covered; and nd = no data. 
plex are not as readily identified as that of $S$. pomi. Perhaps molecular methods will allow rapid and precise identification of these fungi in situ and on spore traps. Probably the development of different SBFS species varies, meaning that developing models that adequately describe inoculum development, fruit infection, and sign development for all fungal species of commercial importance in the complex may be problematic.

In conclusion, development of more accurate SBFS forecast models will depend on several factors. First, collection of meteorological data, particularly wetting and humidity data, will need to be standardized and done in a way that reflects its collection and use in commercial situations. The existence of problems with using LWD as the key forecast variable indicates that other variables, such as RH, temperature, and dew point deficit, should be considered. Additionally, although the development of $S$. pomi on reservoir hosts is reasonably well understood (14), the survival, dispersal, and development of other important fungi in the SBFS complex may need to be better defined in order to develop more effective forecast models. The impacts of different fungicides on these varied species should be determined in order to use fungicides optimally. Finally, differences in tree architecture, orchard sites, and, perhaps, cultivar need to be considered in more effective models. Existing models enable growers to reduce SBFS fungicide applications but may fail $(3,16)$. They predict sign appearance but most do not predict SBFS incidence or severity, or employ an economic threshold, all important concepts in IPM and, hence, valuable to producers making decisions based on expected economic outcomes. Improving SBFS forecasts in the ways suggested here should significantly reduce the frequency of failures and may, perhaps, provide even more savings in fungicide use.

\section{Acknowledgments}

Collaboration among authors and others on this project was supported by a Partnership Grant from the Northeastern IPM Center, United States Department of Agriculture.

\section{Literature Cited}

1. Anonymous. 2005. SpecWare 8 Professional: Disease and Insect Guide. Spectrum Technologies, Inc., Plainfield, IL.

2. Anonymous, 2009. About NEWA. New York State IPM Program Cornell University. http://newa.cornell.edu/index.php?page=about-newa

3. Babadoost, M., McManus, P. S., Helland, S. N., and Gleason, M. L. 2004. Evaluating a wetness-based warning system and reduced-risk fungicides to manage sooty blotch and flyspeck of apple. HortTechnology 14:51-57.

4. Batzer, J. C., Arias, M. M. D., Harrington, T. C., Gleason, M. L., Groenewald, J. Z., and Crous, P. W. 2008. Four species of Zygophiala (Schizothyriaceae, Capnodiales) are associated with the sooty blotch and flyspeck complex on apple. Mycologia 100:246-258.

5. Batzer, J. C., Gleason, M. L., Harrington, T. C., and Tiffany, L. H. 2005. Expansion of the sooty blotch and flyspeck complex on apples based on analysis of ribosomal DNA gene sequences and morphology. Mycologia 97:1268-1286.

6. Batzer, J. C., Gleason, M. L., Taylor, S. E., Koehler, K. J., and Monteiro, J. 2008. Spatial heterogeneity of leaf wetness duration in apple trees and its influence on performance of a warning system for sooty blotch and flyspeck. Plant Dis. 92:164-170.

7. Batzer, J. C., Rincon, S. H., Mueller, D. S., Petersen, B. J., Le Corronc, F., McManus, P. S., Dixon, P. M., and Gleason, M. L. 2010. Effect of temperature and nutrient concentration on the growth of six species of sooty blotch and flyspeck fungi. Phytopathol. Mediterr. 49:3-10.

8. Belding, R. D., Sutton, T. B., Blankenship, S. M., and Young, E. 2000. Relationship between apple fruit epicuticular wax and growth of Peltaster fructicola and Leptodontidium elatius, two fungi that cause sooty blotch disease. Plant Dis. 84:767-772.

9. Biggs, A. R., Cooley, D. R., Rosenberger, D. A., and Yoder, K. 2010. Relative susceptibility of selected apple cultivars to sooty blotch and flyspeck. Plant Health Progress. Online. doi: 10.1094/PHP-2009-1014-01-RS

10. Brown, E. M., and Sutton, T. B. 1993. Time of infection of Gloeodes pomigena and Schizothyrium pomi on apple in North Carolina and potential control by an eradicant spray program. Plant Dis. 77:451-455.

11. Brown, E. M., and Sutton, T. B. 1995. An empirical model for predicting the first symptoms of sooty blotch and flyspeck of apples. Plant Dis. 79:1165-1168

12. Cooley, D. R., Agnello, A. M., and Helms, M., eds. 2010. 2010 New England Tree Fruit Management Guide. New England and Cornell Cooperative Extension Systems, Ithaca, NY.
13. Cooley, D. R., Gamble, J. W., and Autio, W. R. 1997. Summer pruning as method for reducing flyspeck disease on apple fruit. Plant Dis. 81:11231126.

14. Cooley, D. R., Lerner, S. M., and Tuttle, A. F. 2007. Maturation of thyriothecia of Schizothyrium pomi on the reservoir host Rubus allegheniensis. Plant Dis. 91:136-141.

15. Diaz, M. M., Batzer, J. C., Wong, A. A., Bost, S. C., Cooley, D. R., Ellis, M A., Hartman, J. R., Rosenberger, D. A., Sundin, G. W., Sutton, D. K. Travis, J. W., Wheeler, M. J., Yoder, K. S., and Gleason, M. L. 2009. Diversity and biogeography of sooty blotch and flyspeck fungi on apple in the eastern and midwestern United States. Phytopathology 100:345-355.

16. Duttweiler, K. B., Gleason, M. L., Dixon, P. M., Sutton, T. B., McManus, P. S., and Monteiro, J. 2008. Adaptation of an apple sooty blotch and flyspeck warning system for the Upper Midwest United States. Plant Dis. 92:12151222.

17. Ellis, M. A., Madden, L. V., and Wilson, L. 1999. Evaluation of an empirical model for predicting sooty blotch and flyspeck of apples in Ohio. Ohio State Univ. Res. Bull.-Res. Circ. 299-99. Online. http://ohioline.osu. edu/rc299/rc299_4.html

18. Frank, J., Crous, P. W., Groenewald, J. Z., Oertel, B., Hyde, K. D., Phengsintham, P., and Schroers, H. J. 2010. Microcyclospora and Microcyclosporella: novel genera accommodating epiphytic fungi causing sooty blotch on apple. Persoonia 24:93-105.

19. Gadoury, D. M., and MacHardy, W. E. 1982. A model to estimate the maturity of ascospores of Venturia inaequalis. Phytopathology 72:901-904.

20. Gleason, M., Babadoost, M., McManus, P. S., Wegulo, S. N., and Helland, S. J. 2002. Performance of a warning system for sooty blotch and flyspeck on apple using on-site wetness measurements and site-specific wetness estimates. (Abstr.) Phytopathology 92:S29.

21. Gleason, M. L., Batzer, J. C., Sun, G., Zhang, R., Arias, M. M. D., Sutton, T. B., Crous, P. W., Ivanovic, M., McManus, P. S., Cooley, D. R., Mayr, U., Weber, R. W. S., Yoder, K. S., Del Ponte, E. M., Biggs, A. R., and Oertel, B. 2011. A new view of sooty blotch and flyspeck. Plant Dis. 95:368-383.

22. Gleason, M. L., Duttweiler, K. B., Batzer, J. C., Taylor, S. E., Sentelhas, P. C., Monteiro, J., and Gillespie, T. J. 2008. Obtaining weather data for input to crop disease warning systems: leaf wetness duration as a case study. Sci. Agric. 65:76-87.

23. Gleason, M. L., Parker, S. K., Pitblado, R. E., Latin, R. X., Speranzini, D. Hazzard, R. V., Maletta, M. J., Cowgill, W. P., and Biederstedt, D. L. 1997. Validation of a commercial system for remote estimation of wetness duration. Plant Dis. 81:825-829.

24. Grabowski, M., and Wrona, B. 2004. An investigation of the date of sooty blotch primary infection and duration of incubation period for selected apple cultivars. Folia Hortic. 16:73-77.

25. Hartman, J. R. 1995. Evaluation of fungicide timing for sooty blotch and flyspeck control, 1994. Fungic. Nematicide Tests 50:10.

26. Hickey, K. D. 1960. The sooty blotch and flyspeck diseases of apple with emphasis on variation within Gloeodes pomigena (Schw.) Colby. Ph.D. dissertation, The Pennsylvania State University, State College.

27. Hickey, K. D., Lewis, F. H., and Taylor, C. F. 1958. Time of apple fruit infection by Gloedes pomigena and Microthyriella rubi. Phytopathology 48:462-462.

28. Huber, L., and Gillespie, T. J. 1992. Modeling leaf wetness in relation to plant disease epidemiology. Annu. Rev. Phytopathol. 30:553-577.

29. Johnson, E. M., and Sutton, T. B. 2000. Response of two fungi in the apple sooty blotch complex to temperature and relative humidity. Phytopathology 90:362-367.

30. Jones, A. L., and Sutton, T. B. 1996. Diseases of Tree Fruits in the East: Mich. State Univ. Coop. Ext. NCR 045, East Lansing.

31. Kelley, J. G. W., Russo, J. M., Eyton, J. R., and Carlson, T. N. 1988 Mesoscale forecasts generated from operational numerical weather-prediction model output. Bull. Am. Meteorol. Soc. 69:7-15.

32. Kim, K. S., Gleason, M. L., and Taylor, S. E. 2006. Forecasting site-specific leaf wetness duration for input to disease-warning systems. Plant Dis. 90:650-656.

33. Koehler, G. 2007. Introduction to orchard radar. University of Maine http://pronewengland.org/allmodels/RadarIntro.htm

34. Lau, Y. F., Gleason, M. L., Zriba, N., Taylor, S. E., and Hinz, P. N. 2000 Effects of coating, deployment angle, and compass orientation on performance of electronic wetness sensors during dew periods. Plant Dis. 84:192197.

35. Li, H. Y., Zhang, R., Sun, G. Y., Batzer, J. C., and Gleason, M. L. 2010. New species and record of Zygophiala on apple fruit from China. Mycol. Prog. 9:245-251.

36. MacHardy, W. E., and Gadoury, D. M. 1989. A revision of Mills criteria for predicting apple scab infection periods. Phytopathology 79:304-310.

37. Magarey, R. D., Russo, J. M., Seem, R. C., and Gadoury, D. M. 2005. Surface wetness duration under controlled environmental conditions. Agric. For. Meteorol. 128:111-122.

38. Magarey, R. D., Seem, R. C., and Russo, J. M. 2006. Grape canopy surface wetness: simulation versus visualization and measurement. Agric. For Meteorol. 139:361-372.

39. Magarey, R. D., Seem, R. C., Russo, J. M., Zack, J. W., Waight, K. T., Travis, J. W., and Oudemans, P. V. 2001. Site-specific weather information 
without on-site sensors. Plant Dis. 85:1216-1226.

40. Ocamb-Basu, C. M., and Sutton, T. B. 1988. Effects of temperature and relative-humidity on germination, growth, and sporulation of Zygophiala jamaicensis. Phytopathology 78:100-103.

41. Rosenberger, D. A. 1994. Summer disease control in apples. Pages 80-86 in: 124th Annu. Rep. State Hortic. Soc. Mich.

42. Rosenberger, D. A., and Meyer, F. W. 2006. Post-infection control of flyspeck with new fungicides, 2005. Fungic. Nematicide Tests 61. Online. doi: 10.1094/FN61

43. Rosenberger, D. A., and Meyer, F. W. 2007. Timing summer fungicides to control flyspeck disease on apples. N. Y. Fruit Q. 15:11-14.

44. Rosenberger, D. A., Meyer, F. W., Ahlers, C. A., and VanCamp, K. L. 2002. Post-infection activity of Sovran, Flint, Benlate, and Topsin M for control of flyspeck and sooty blotch, 2001. Fungic. Nematicide Tests 57. Online. doi: 10.1094/FN57

45. Rosenberger, D. A., Meyer, F. W., and Christiana, R. W. 2005. Control of summer diseases with Topsin M, Captan, strobilurins, and copper fungicides, 2004. Fungic. Nematicide Tests 60. Online. doi:10.1094/FN60

46. Rosenberger, D. A., Meyer, F. W., and Christiana, R. W. 2005. Summer disease control with Captan, Flint, Pristine, Sovran and Topsin M, 2004. Fungic. Nematicide Tests 60. Online. doi: 10.1094/FN60

47. Russo, J. M. 2000. Weather forecasting for IPM. Pages 453-473 in: Emerging Techniques for Integrated Pest Management: Concepts, Research, and Implementation. G. G. Kennedy and T. B. Sutton, eds. American Phytopathological Society, St. Paul, MN.

48. Sentelhas, P. C., Gillespie, T. J., Batzer, J. C., Gleason, M. L., Monteiro, J., Pezzopane, J. R. M., and Pedro, M. J. 2005. Spatial variability of leaf wetness duration in different crop canopies. Int. J. Biometerol. 49:363-370.

49. Smigell, C. G., and Hartman, J. R. 1997. Evaluation of fungicide timing for sooty blotch and flyspeck control, 1996. Fungic. Nematicide Tests 52:31.

50. Smigell, C. G., and Hartman, J. R. 1998. Evaluation of multi-layer fruit bags for cork spot, sooty blotch and flyspeck control, 1997. Biol. Cult. Tests $13: 39$
51. Stensvand, A., Eikemo, H., Gadoury, D. M., and Seem, R. C. 2005. Use of a rainfall frequency threshold to adjust a degree-day model of ascospore maturity of Venturia inaequalis. Plant Dis. 89:198-202.

52. Stensvand, A., Gadoury, D. M., Amundsen, T., Semb, L., and Seem, R. C. 1997. Ascospore release and infection of apple leaves by conidia and ascospores of Venturia inaequalis at low temperatures. Phytopathology 87:10461053.

53. Sutton, J. C., Gillespie, T. J., and Hildebrand, P. D. 1984. Monitoring weather factors in relation to plant disease. Plant Dis. 68:78-84.

54. Sutton, T. B. 1996. Changing options for the control of deciduous fruit tree diseases. Annu. Rev. Phytopathol. 34:527-547.

55. Trapman, M. 2004. A simulation program for the timing of fungicides to control sooty blotch in organic apple growing. First results in 2003. Paper presented at 11th Int. Conf. Cultivation Technique and Phytopathological Problems in Organic Fruit-Growing, Weinsberg, Germany. Online. http://orgprints.org/14068/

56. Trapman, M. 2006. Observations on the epidemiology of sooty blotch in organic orchards in the Netherlands. Paper presented at 12th Int. Conf. Cultivation Technique and Phytopathological Problems in Organic FruitGrowing, Weinsberg, Germany. Online. http://orgprints.org/8841/

57. Tuttle, A. F., Bergweiler, C., Hall, J., Reisner, L., Christle, S., Autio, W. R., and Cooley, D. R. 2002. Development of a model for predicting flyspeck risks in blocks of apple trees. Fruit Notes. 67 (1). Online. http://www.umass.edu/fruitadvisor/fruitnotes/developmentofa.pdf

58. Williamson, S. M., and Sutton, T. B. 2000. Sooty blotch and flyspeck of apple: etiology, biology, and control. Plant Dis. 84:714-724.

59. Wrona, B., and Grabowski, M. 2004. Influence of fructose and glucose occurring on fruit surface on the growth of fungi that cause sooty blotch of apple. J. Plant Prot. Res. 44:287-291.

60. Yang, H. L., Sun, G. Y., Batzer, J. C., Crous, P. W., Groenewald, J. Z., and Gleason, M. L. 2010. Novel fungal genera and species associated with the sooty blotch and flyspeck complex on apple in China and the USA. Persoonia 24:29-37. 